\title{
Zanamivir oral delivery: possibilities revisited
}

"...the ability of zanamivir to reach the lung following systemic availability is out of concern for the formulators as demonstrated by preclinical and clinical studies with oral or intravenous zanamivir administration."

Keywords: influenza • neuraminidase inhibitor • oral bioavailability • zanamivir

Influenza is a highly contagious respiratory tract infection caused by influenza type $\mathrm{A}$ and $B$ viruses. Influenza can cause serious morbidity and increased mortality in certain special populations such as the elderly, the immunocompromised and individuals with underlying diseases such as chronic obstructive pulmonary disease, cardiovascular disease and diabetes [1]. Until now, only five drugs have been approved by US FDA for the treatment of influenza infections: amantadine, rimantadine, zanamivir, oseltamivir and peramivir. Of these, amantadine and rimantadine belong to a class of ion channel inhibitors that act by interfering with the viral coating process and are only effective against influenza $\mathrm{A}$. The use of this class of drugs has reduced due to a rapid increase in resistance to influenza $\mathrm{A}$. However, zanamivir, oseltamivir and peramivir belong to a class of neuraminidase inhibitors that prevent viral propagation by inhibiting the neuraminidase enzyme necessary for viral propagation and are potent inhibitors of both influenza A and influenza B. Among these neuraminidase inhibitors, peramivir has been approved recently (December 2014) and reports on peramivir-related resistance are not available yet. However, there are many reported cases of oseltamivir-related resistance in the patient population since its clinical use [2]. Nevertheless, there are no reports of resistance to zanamivir to date due to its structural similarity with sialic acid, which makes it a drug of choice in the treatment of influenza including oseltamivir-resistant variants as well as other emerging resistant strains in the prevention and treatment of seasonal and pandemic influenza.
Currently, zanamivir is available as Relenza ${ }^{\circledR}$ for oral inhalation, which is a dry powder formulation intended for inhalation using Diskhaler ${ }^{\circledR}$ (a dry powder inhaler). The predominant disadvantages with this route of administration are lack of patient compliance and difficulty of drug administration to the pediatric population which is at increased risk of influenza infection. Additionally, it is recommended against for some chronic respiratory conditions. Until now, no formulation of zanamivir for other routes of administration, such as parenteral or oral, has been available. Recently, a myriad of clinical studies established the efficacy and safety of intravenous zanamivir $[3,4]$ and development of intravenous formulation of zanamivir is being actively pursued by the drug sponsor (GSK).

This new prospect has also thrown an opportunity for avid drug delivery scientists and formulators to revisit the possibility of oral delivery of zanamivir. An oral formulation is preferred due to its several advantages over intravenous administration. In addition to the flexibility of treatment, oral formulations are inexpensive, convenient and have higher rate of patient compliance.

Unfortunately, oral formulation of zanamivir is not available currently due to its poor oral bioavailability (BA); $2 \%$ (range $1-5 \%$ ) in humans [1]. The poor BA of zanamivir could be attributable to its tremendously high hydrophilicity resulting from the polar and zwitterionic nature of its molecule. According to Lipinski's rule of five that is useful in the assessment of drugability or drug likeness, the violation score for zanamivir is 2

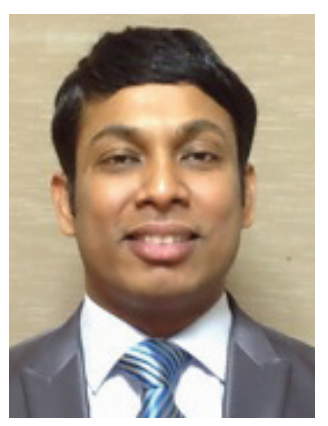

Srinivasan Shanmugam Pharm. R\&D Institute, Hanmi Pharm. Co., Ltd., Hwasung, Gyeonggi 445-913, Korea Tel.: +82 313563311 Fax: +82313567139 rxsrinivasan@gmail.com 
(range of violation score is $0-4$, with 0 being 'good absorption/permeation' and 4 being 'poor absorption/ permeation') with hydrogen bond donors of seven, hydrogen bond acceptors of 10 , molecular weight of 332.3 and $\operatorname{cLog} \mathrm{P}$ of -4.20 . Besides, the topological polar surface area, polar surface area and apolar surface area of zanamivir was 200.73, 291.60 and 230.70, respectively with apolar surface area to polar surface area ratio of 0.79 [5]. Most of these properties indicate a low bioavailability of zanamivir in vivo and positions the drug in biopharmaceutics classification system Class III with high solubility and poor permeability.

There is scarce availability of preclinical or clinical reports on the oral BA of zanamivir, not to mention the attempts to enhance the oral BA of zanamivir. One of the clinical Phase I studies (open-label, single-dose, five-way crossover study) by GSK (Clinical Study ID NAI107485) to examine the relative oral bioavailability of zanamivir with bioenhancing excipients $(200 \mathrm{mg}$ zanamivir and $600 \mathrm{mg}$ excipient) following direct release into mid-small intestine using $\gamma$-scintigraphy and the InteliSite ${ }^{\circledR}$ companion capsule in healthy subjects demonstrated about fivefold increase in AUC and about 11-fold increase in $\mathrm{C}_{\max }$ compared with control zanamivir at a dose of $200 \mathrm{mg}$ [19].

Since then, very few attempts were made to enhance the oral bioavailability of zanamivir. Prodrug approaches have been investigated by Miller et al., Gupta $e t a l$. and Liu et al. [6-8] and intestinal permeability enhancer technique by Shanmugam et al. and Holmes et al. [9,10]. Additionally, lipid formulations such as liposomes by Boonyapiwat et al., and solid lipid nanoparticles by Cao et al. and Shi et al., have also been explored [11-13]. All studies demonstrated enhanced oral BA of zanamivir except the solid lipid nanoparticles. Besides enhanced BA, reachability or availability of zanamivir in the target site (lung) following systemic availability is one of the major factors for achieving efficacy. Interestingly, recent preclinical and clinical studies corroborated the lung reachability or availability of zanamivir from systemic circulation following oral or intravenous administration [9,14-17]. Volume of distribution and protein binding could also serve as reasonable means to understand the possibility of any drug availability in extravascular tissues and other organs. The steady-state volume of distribution and percentage protein binding for zanamivir is 161 and $<10 \%$, respectively [18]. Since the unbound drug is mostly available for passive diffusion into the extravascular site or tissue, it could be possible that the lung availability or reachability of zanamivir from plasma or systemic circulation would be similar or higher than plasma concentration as the steady-state volume of distribution of zanamvir is higher and approximates the extracellular water. There is no reported first pass effect or liver metabolism of zanamivir, which is advantageous in case of zanamivir that has poor bioavailability. Assuming that bioavailability enhancement is achieved and a significant portion of the drug reaches the target organ, it is of paramount importance to know the target concentration for efficacy.

Typically, the Diskhaler releases around $90 \%$ of the dosage form into the respiratory tract, leaving about $10 \%$ of drug retained in the blister and device. The pharmacological availability of zanamivir that reaches the lung is less than $15 \%$ (13.2\% in the bronchi and lungs and $1.2 \%$ in trachea) of the total dose, which is less than $1.5 \mathrm{mg}$ of the $10 \mathrm{mg}$ dose. The rest of the dose (about 77.6\%) is deposited on the oropharynx and is eliminated immediately by passage through the gastrointestinal tract [16]. The percentage of it that dissolves into lung secretions to produce the desired effect without agglomeration is questionable. Even at this amount of presence in the lung, the zanamivir concentrations were reported to be more than $10 \mu \mathrm{mol} / \mathrm{l}$ which is significantly higher than $\mathrm{IC}_{50}$ concentration of $0.64-7.9 \mathrm{nmol} / \mathrm{l}$ for influenza viruses. This local concentration of zanamivir following oral inhalation is about 15,625 to 1262 times higher than the $\mathrm{IC}_{50}$ concentrations of influenza viruses [16]. Now, a series of question arises such as, does zanamivir need to be presented in such a high concentration to produce necessary effect? Does this high concentration provide any additional beneficial effects in terms of efficacy and/ or resistance? And, finally and most importantly, how much concentration would be appropriate for efficacy and/or prevention of resistance, especially if oral formulation is intended?

Although targeting zanamivir concentrations of more than the $\mathrm{IC}_{50}$ concentration of $0.64-7.9 \mathrm{nmol} / \mathrm{l}$ seems to be a good bet to begin with, antiviral effect of zanamivir is usually achieved by saturating the sialidase enzymes and thus preventing the mobility of virus particles through the respiratory tract mucus and the elution of virion progeny from the infected cells. In the case of inhaled zanamivir formulation, high local concentrations of inhibitor (zanamivir) are achieved at the surface of infected cells, resulting in high efficacy due to higher levels of inhibitor binding to the enzyme. Similarly, for zanamivir oral formulations, achievement of a high $\mathrm{C}_{\max }$ (maximum plasma concentration) capable of saturating the sialidase enzyme at the site of action is necessary for efficacy. Thus, the primary concern during oral formulation development should be enhancing $\mathrm{C}_{\max }$ to the highest possible level and eventually optimizing the dose based on the efficacy. Since $600 \mathrm{mg}$ intravenous zanamivir twice daily is nontoxic and well-tolerated $[3,4]$, it is possible that oral 
dose of $600 \mathrm{mg}$ or higher could be explored to achieve the desired oral bioavailability and efficacy.

In conclusion, the ability of zanamivir to reach the lung following systemic availability is out of concern for the formulators as demonstrated by preclinical and clinical studies with oral or intravenous zanamivir administration. Nonetheless, the ultimate task for the formulators is to achieve a high $\mathrm{C}_{\max }$ capable of saturating the sialidase enzyme at the site of action for efficacy, regardless of the chemical, prodrug or pharmaceutical formulation approaches. Considering various available and ongoing clinical studies on efficacy and safety of the intravenous zanamivir by GSK and other research groups, it is conceivable that the commercial intravenous

\section{References}

1 Elliott M. Zanamivir: from drug design to the clinic. Phil. Trans. R. Soc. B. 356(1416), 1885-1893 (2001).

2 De Jong MD, Tran TT, Truong HK et al. Oseltamivir resistance during treatment of influenza $\mathrm{A}(\mathrm{H} 5 \mathrm{~N} 1)$ infection. N. Engl. J. Med. 353(25), 2667-2672 (2005).

3 Watanabe A, Yates PJ, Murayama M, Soutome T, Furukawa H. Evaluation of safety and efficacy of intravenous zanamivir in the treatment of hospitalized Japanese patients with influenza: an open-label, single-arm study. Antivir. Ther. doi:10.3851/IMP2922. (2014) (Epub ahead of print).

4 Marty FM, Man CY, Van Der Horst C et al. Safety and pharmacokinetics of intravenous zanamivir treatment in hospitalized adults with influenza: an open-label, multicenter, single-arm, phase II study. J. Infect. Dis. 209(4), 542-550 (2014).

5 Heneghan CJ, Onakpoya I, Thompson M, Spencer EA, Jones M, Jefferson T. Zanamivir for influenza in adults and children: systematic review of clinical study reports and summary of regulatory comments. BMJ 348, g2547 (2014).

6 Miller JM, Dahan A, Gupta D, Varghese S, Amidon GL. Enabling the intestinal absorption of highly polar antiviral agents: ion-pair facilitated membrane permeation of zanamivir heptyl ester and guanidino oseltamivir. Mol. Pharm. 7(4), 1223-1234 (2010).

7 Gupta SV, Gupta D, Sun J et al. Enhancing the intestinal membrane permeability of zanamivir: a carrier mediated prodrug approach. Mol. Pharm. 8(6), 2358-2367 (2011).

$8 \quad$ Liu KC, Lee PS, Wang SY, Cheng YS, Fang JM, Wong $\mathrm{CH}$. Intramolecular ion-pair prodrugs of zanamivir and guanidino-oseltamivir. Bioorg. Med. Chem. 19(16), 4796-4802 (2011).

9 Shanmugam S, Im HT, Sohn YT et al. Zanamivir oral delivery: enhanced plasma and lung bioavailability in rats. Biomol. Ther. 21(2), 161-169 (2013).

10 Holmes EH, Devalapally H, Li L, Perdue ML, Ostrander GK. Permeability enhancers dramatically increase zanamivir formulation is not far away. However, given the necessity of oral dosage form of zanamivir and possibilities of oral BA enhancement, more intensive investigations are warranted for oral delivery of zanamivir.

\section{Financial \& competing interests disclosure}

The authors have no relevant affiliations or financial involvement with any organization or entity with a financial interest in or financial conflict with the subject matter or materials discussed in the manuscript. This includes employment, consultancies, honoraria, stock ownership or options, expert testimony, grants or patents received or pending, or royalties.

No writing assistance was utilized in the production of this manuscript.

absolute bioavailability in rats: implications for an orally bioavailable influenza treatment. PLoS ONE 8(4), e61853 (2013).

11 Boonyapiwat B, Sarisuta N, Kunastitchai S. Characterization and in vitro evaluation of intestinal absorption of liposomes encapsulating zanamivir. Curr. Drug Deliv. 8(4), 392-297 (2011).

12 Cao Q, Wu H, Zhu L et al. Preparation and evaluation of zanamivir-loaded solid lipid nanoparticles. J. Control. Release 152(Suppl. 1), e2-e4 (2011).

13 Shi LL, Cao Y, Zhu XY, Cui JH, Cao QR. Optimization of process variables of zanamivir-loaded solid lipid nanoparticles and the prediction of their cellular transport in Caco-2 cell model. Int. J. Pharm. 478(1), 60-69 (2014).

14 Shelton MJ, Lovern M, Ng-Cashin J et al. Zanamivir pharmacokinetics and pulmonary penetration into epithelial lining fluid following intravenous or oral inhaled administration to healthy adult subjects. Antimicrob. Agents Chemother. 55(11), 5178-5184 (2011).

15 Cass LM, Efthymiopoulos C, Bye A. Pharmacokinetics of zanamivir after intravenous, oral, inhaled or intranasal administration to healthy volunteers. Clin. Pharmacokinet. 36(Suppl. 1), 1-11 (1999).

16 Cass LM, Brown J, Pickford M et al. Pharmacoscintigraphic evaluation of lung deposition of inhaled zanamivir in healthy volunteers. Clin. Pharmacokinet. 36(Suppl. 1), 21-31 (1999).

17 Sidwell RW, Huffman JH, Barnard DL et al. Inhibition of influenza virus infections in mice by GS4104, an orally effective influenza virus neuraminidase inhibitor. Antivir. Res. 37(2), 107-120 (1998).

18 Pukrittayakamee S, Jittamala P, Stepniewska K et al. An open-label crossover study to evaluate potential pharmacokinetic interactions between oral oseltamivir and intravenous zanamivir in healthy Thai adults. Antimicrob. Agents Chemother. 55(9), 4050-4057 (2011).

19 GSK Clinical Study Register www.gsk-clinicalstudyregister.com 